complications. LGIE contributed to the final diagnosis of gastrointestinal disorder in 102/210 (48.6\%) by showing histological findings in favor of the diagnosis in biopsies or negative signs allowing to eliminate the diagnosis or by revealing abnormalities in proctologic examination.

Conclusion Rates of endoscopic and histological abnormalities from LGIE vary based on age and indication for endoscopy. The diagnostic yield of LGIE was moderate (39\%). However, LGIE contributed to diagnosis and management of patients in about half of the cases (48.6\%). Negative findings from LGIE can contribute in a positive way to the diagnosis and management of children with gastrointestinal symptoms. A study must be conducted to identify factors associated with diagnostic yield of LGIE in children.

\section{P331 EXTRAINTESTINAL MANIFESTATIONS OF PEDIATRIC INFLAMMATORY BOWEL DISEASE: A TUNISIAN SINGLE- CENTER EXPERIENCE}

\begin{abstract}
${ }^{1,2}$ Rania Ben Rabeh* ${ }^{1,2}$ Asma Ben Othman, 1,2 Olfa Bouyahya, ${ }^{1,2}$ Sonia Mazigh Mrad, ${ }^{1,2}$ Samir Boukthir. 'Pediatric Gastroenterology Department, Bechir Hamza Children's Hospital , Tunis, Tunisia; ${ }^{2}$ University Tunis El Manar, Faculty of medicine, Tunis, Tunisia
\end{abstract}

\subsection{6/archdischild-2019-epa.680}

Introduction The incidence of pediatric inflammatory bowel disease (IBD) increased during last years. However, extra intestinal manifestations (EIM) in children with IBD are poorly characterized. The aim of this study was to describe clinical features of extraintestinal manifestations of IBDs in the Tunisian pediatric population.

Methods We conducted a retrospective study from 2012 to 2017 of children admitted to the pediatric gastroenterology department of BECHIR HAMZA Children's Hospital for Crohn's disease (CD) or ulcerative colitis (UC).

Results We collected 14 patients, six boys and eight girls, five cases with UC and nine cases with $\mathrm{CD}$. The mean age at diagnosis was $10 \pm 3.3$ years [18 month-14 years]. EIMs were reported in ten of 14 patients. EIMs included aphthous stomatitis $(n=3)$, osteoporosis/osteopenia $(n=4)$, peripheral joint infammation $(n=5)$, primary sclerosing cholangitis $(n=1)$, ankylosing spondylitis $(n=1)$, cerebral venous thrombosis $(n=1)$ and cerebral vasculitis $(n=1)$. We observed three children with skin involvement : one with erythema nodosum, the other with ulcerative skin eruption and a case of vitiligo. EIMs were more frequent in CD than UC (7/9 vs $3 / 5, \mathrm{p}=$ $0.041)$. EIM appeared before IBD diagnosis in $11 / 18$ cases. Three patients were treated with anti-TNF agents, six with azathioprine and only one with methotrexate.

Conclusion The prevalence of EIMs in children with IBD in our study was high mainly in patients with CD. EIM may appear before IBD diagnosis. Knowledge of these fndings may led to an increased awareness of underlying IBD, thereby decreasing diagnostic delay.

\section{P332 COMPLICATIONS OF ACHALASIA IN A PAEDIATRIC PATIENT WITH DOWN SYNDROME}

${ }^{1}$ Ana-Georgeta Galatan*, ${ }^{2}$ Constantin Ciuce, ${ }^{1,2}$ Alexandru Pirvan. ${ }^{1}$ Clinical Emergency Hospital for Children, Cluj-Napoca, Romania; ${ }^{2}$ University of Medicine and Pharmacy 'Iuliu Hatieganu', Cluj-Napoca, Romania

10.1136/archdischild-2019-epa.681
Introduction Achalasia is a motility disorder whose pathophysiology is still incompletely understood. Although rare, achalasia can be associated with Down syndrome, with a higher prevalence than the general population. The treatment is palliative and the medical management often fails whereas the endoscopic and surgical treatment relief symptoms on the longterm with comparable success rates.

Case presentation We herein report the case of a 12 years old girl who was first referred to our hospital at the age of 3 for severe growth retardation. She was diagnosed from birth with Down syndrome by translocation, ventricular septal defect and interventricular membranous septal aneurysm. She presented post-prandial regurgitation from infancy, failure to thrive and numerous respiratory tract infections. Based on clinical symptoms and timed barium oesophagogram we defined the case as achalasia with megaoesophagus. She was transferred to the surgical unit and underwent a surgical myotomy with fundoplication. She developed an oesophageal-mediastinal fistula for which a total oesophagectomy was performed, with colon interposition for oesophageal replacement. One year later, the patient presented melena and required another surgery for cologastric anastomotic stricture and anastomotic ulcerations. After a long-term asymptomatic period, during her last assessment she was diagnosed with pneumonia, aspiration syndrome and pleural effusion. The CT scan described a dilated, tortuous colon graph with significant stasis. As there was an important intra-thoracic compression with respiratory distress, she was transferred for surgical treatment considering this lifethreatening disorder.

Discussion This case highlights the complex treatment of an uncommon association of achalasia and Down syndrome by translocation. Given that the treatment for achalasia in children is still continuously debated, the therapeutic option should depend on the patient and further attention should be given towards the long-term complications .

\section{P333 AN UNUSUAL CASE- ASCITES IN A TWO YEAR OLD CHILD}

Ciara Lane*, Michael O’Neill. Mayo University Hospital, Mayo, Ireland

\subsection{6/archdischild-2019-epa.682}

Aims Ascites in the paediatric population is very rare with no known prevalence. This case of ascites secondary to portal vein thrombosis demonstrates the work up required and complications that can occur.

Methods DH a previously healthy 2 year old boy presented with 3 week history of increased abdominal distention. $\mathrm{He}$ was clinically well with no anorexia, dyspnoea or discomfort. He had a normal urine output and bowel habit. No past medical history of note. On examination, he was a happy, well child with normal hydration status. His weight was $14.7 \mathrm{~kg}$ (91st-99th centile). His abdominal exam revealed marked distention; umbilicus everted \& enlarged abdominal girth of 60 $\mathrm{cm}$. Tense ascites present with positive fluid thrill. His bloods, including coagulation screen and liver function tests, were all normal. Ultrasound scan of liver showed cavernous transformation of left portal vein, splenomegaly and large volume ascites.

Results $\mathrm{He}$ was diagnosed with a portal vein thrombosis (PVT), this is a cause of ascites in 6 per 1,000 children $^{[1]}$. The PVT aetiology was investigated analysing for intrinsic \& 